\title{
Thrombotic Thrombocytopenic Purpura, CTCAE
}

National Cancer Institute

\section{Source}

National Cancer Institute. Thrombotic Thrombocytopenic Purpura, CT CAE. NCI

Thesaurus. Code C143874.

A disorder characterized by the presence of microangiopathic hemolytic anemia,

thrombocytopenic purpura, fever, renal abnormalities and neurological abnormalities

such as seizures, hemiplegia, and visual disturbances. It is an acute or subacute condition. 